\title{
Sincronização Parcial em uma Rede de Populações Acopladas
}

\author{
Flávia T. Giordani, \\ Departamento de Matemática, UFSC, \\ CEP 88040-900, Florianópolis, SC \\ E-mail: flavia.giordani@ufsc.br,
}

\begin{abstract}
Resumo: Neste trabalho investigamos a ocorrência de sincronização parcial em uma rede de populaçôes acopladas de múltiplas espécies. Através de evidências numéricas, analisamos os efeitos da dispersão não linear na formação de clusters, considerando a região de impossibilidade de coerência.
\end{abstract}

Palavras-chave: Sincronização parcial, metapopulação, números de Liapunov.

\section{Introdução}

Órbitas sincronizadas em redes de populações acopladas correspondem àquelas em que as densidades populacionais em cada sítio evoluem no tempo com a mesma amplitude e fase (dinâmica coerente [9]). A importância desse fenômeno para o estudo de sistemas metapopulacionais tem sido destacada de forma intensa na literatura, haja visto sua relação com persistência e extinção de espécies e com a ocorrência de recolonização [6]. Com isso, para certos parâmetros do sistema, critérios para a estabilidade de órbitas coerentes tem sido obtidos [4],[5]. No entanto, para valores dos parâmetros onde a ocorrência de sincronização é impossível, a dinâmica global pode ser bastante complexa e o fenômeno da sincronização parcial pode ocorrer. Nessa situação, ao invés de todos os sítios estarem sincronizados, um ou mais grupos de sítios entram em sincronia, formando os denominados "clusters". Em termos práticos, o estudo da sincronização parcial pode ser relevante, por exemplo, em sistemas ecológicos de múltiplas espécies coexistindo; empiricamente, a existência de múltiplos atratores caóticos em sincronia pode indicar conservação ou extinção dessas espécies [8],[3].

No presente trabalho analisamos uma rede acoplada de populações com múltiplas espécies e migração dependente da densidade. Diversos trabalhos tem mostrado que a dispersão não linear em mapas acoplados pode favorecer a sincronia [7], [11]. Em [5], essa análise foi realizada para um modelo populacional de múltiplas espécies e um critério de estabilidade transversal para atratores sincronizados foi estabelecido, baseado nos números transversais de Liapunov. No entanto, há um rico espectro de possibilidades na região de impossibilidade de coerência e é exatamente nessa região que nos concentramos nesse trabalho, investigando a ocorrência de sincronização parcial e sua relação com a função de migração dependente das densidades locais. Nossa análise é essencialmente numérica, haja visto que ainda não desenvolvemos critérios de estabilidade assintótica para atratores contidos em subvariedades invariantes do espaço de fase em estado "clusterizado" (para mapas linearmente e globalmente acoplados tais critérios são estabelecidos em [10],[3]). Nesse sentido, apresentamos várias simulações numéricas que evidenciam a influência da migração dependente da densidade em um modelo metapopulacional de duas espécies com hierarquia na dinâmica local. 


\section{O Modelo}

Consideramos $n$ sítios enumerados de 1 até $n$. Em cada sítio existe uma comunidade de $k$ espécies que chamamos de população local (subpopulação). O vetor populacional no sítio $j$ no tempo $t$ é $\mathbf{x}_{j}^{t}=\left(x_{1 j}^{t}, x_{2 j}^{t}, \ldots, x_{k j}^{t}\right)^{T} \in \mathbb{R}^{k}$ onde $x_{\ell j}^{t}$ representa densidade de indivíduos da espécie $\ell$ no sítio $j$ no tempo $t$. Supomos que a cada geração os indivíduos passam por dois processos distintos: o processo de dinâmica local, que corresponde a reprodução e sobrevivência das espécies, e o processo de migração. O primeiro é descrito pela equação

$$
\mathbf{x}_{j}^{t+1}=\mathbf{f}\left(\mathbf{x}_{j}^{t}\right), \quad \forall j=1,2, \ldots, n, t=0,1,2, \ldots,
$$

em que $\mathbf{f}: \mathbb{R}^{k} \rightarrow \mathbb{R}^{k}$ é suave. O segundo processo ocorre quando são estabelecidas conexões entre os sítios, ou seja, há a possibilidade dos indivíduos de cada espécie migrarem para outros sítios. Para descrever tal processo, denotamos $c_{j i}$ como sendo a proporção de indivíduos que migra do sítio $i$ para o sítio $j$ e $\mu_{i}: \mathbb{R}^{k} \rightarrow \mathbb{R}$ uma função suave representando a fração de migração da espécie $i$, ou seja, a proporção dos indivíduos da espécie $i$ que deixam um certo sítio. Admitindo que a dinâmica local precede o processo de migração, ou seja, em cada geração, após o processo de dinâmica local, uma fração $\mu_{i}$ de indivíduos da espécie $i$ deixa um dado sítio e migra para os sítios mais próximos, a dinâmica da metapopulação é dada por

$$
\mathbf{x}_{j}^{t+1}=\mathbf{f}\left(\mathbf{x}_{j}^{t}\right)-M\left(\mathbf{f}\left(\mathbf{x}_{j}^{t}\right)\right) \mathbf{f}\left(\mathbf{x}_{j}^{t}\right)+\sum_{i=1}^{n} c_{j i} M\left(\mathbf{f}\left(\mathbf{x}_{i}^{t}\right)\right) \mathbf{f}\left(\mathbf{x}_{i}^{t}\right), t=0,1,2, \ldots,, j=1,2, \ldots, n,
$$

onde $M(\mathbf{x})=\operatorname{diag}\left(\mu_{1}(\mathbf{x}), \mu_{2}(\mathbf{x}), \ldots, \mu_{k}(\mathbf{x})\right)$ (para detalhes ver [5]). Vamos denotar também $C:=\left(c_{i j}\right), B:=I_{n}-C$ e $\Phi(\mathbf{x})=M(\mathbf{x}) \mathbf{x}$.

Uma órbita do sistema (2) é dita estar em estado sincronizado se, para cada $t=0,1,2, \ldots$, temos $\mathbf{x}_{i}^{t}=\mathbf{x}_{j}^{t} \equiv \mathbf{x}_{s}^{t}, \forall i, j=1,2, \ldots, n$. A estabilidade assintótica de atratores sincronizados pode ser obtida através de uma linearização adequada do sistema e da evolução de uma perturbação a órbitas sincronizadas. Assim, considerando somente perturbações transversais ao atrator, este será assintoticamente (transversalmente) estável se $L_{T}:=\lim _{\tau \rightarrow+\infty}\left\|P^{\tau-1} P^{\tau-2} \ldots P^{0}\right\|^{\frac{1}{\tau}}<1$, onde $P^{\tau}=\left(I_{n-1} \otimes D \mathbf{f}\left(\mathbf{x}_{s}^{\tau}\right)-A^{\tau} \otimes\left(D \Phi^{\tau}\left(\mathbf{f}\left(\mathbf{x}_{s}^{\tau}\right)\right) D \mathbf{f}\left(\mathbf{x}_{s}^{\tau}\right)\right)\right)$, $A$ é uma matriz obtida através da decomposição do sistema em componentes transversal e paralela à subvariedade invariante que contém o atrator e $\otimes$ é o produto de Kronecker. O limite $L_{T}$ corresponde ao maior número transversal de Liapunov do atrator, sendo assim, a região onde coerência é impossível ocorre quando $L_{T}>1$.

\section{Sincronização Parcial - Resultados Numéricos}

Em [3] observou-se, para um modelo metapopulacional com migração não dependente da densidade, que fenômenos complexos tais como sincronização parcial, intermitência on-off e bifurcação blow-out podem ocorrer na região de impossibilidade de coerência. Isso sugere que a dinâmica de modelos com acoplamento não linear pode ser ainda mais imprevisível. Nosso interesse é investigar essa dinâmica no caso do sistema (2), para isso diremos que uma espécie $i$ do sistema está parcialmente sincronizada se existirem sítios $n_{1}, n_{2}, \ldots, n_{J}$ tais que $x_{i n_{1}}^{t}=x_{i n_{2}}^{t}=\ldots=x_{i n_{J}}^{t}$, para todo $t=0,1,2, \ldots$. Os sítios $n_{1}, \ldots, n_{J}$ formam um cluster para a espécie $i$ e, como veremos a seguir, para uma mesma espécie podem existir vários clusters. Consideremos o seguinte modelo hierárquico de duas espécies,

$$
\left\{\begin{array}{l}
x_{1}^{t+1}=x_{1}^{t} e^{r_{1}\left(1-x_{1}^{t}\right)} \\
x_{2}^{t+1}=x_{2}^{t} e^{r_{2}\left(1-\alpha x_{1}^{t}-x_{2}^{t}\right)},
\end{array}\right.
$$

onde $x_{1}^{t}$ representa a densidade populacional da espécie 1 no tempo $t$ e $x_{2}^{t}$ a densidade populacional da espécie 2 no tempo $t$. Os parâmetros $r_{1}>0$ e $r_{2}>0$ correspondem às taxas 
de crescimento intrínseco das espécies 1 e 2 respectivamente, e $0<\alpha<1$ mede os efeitos de competitividade da espécie $1 \mathrm{com}$ a espécie 2 (ver [2]). A fração migratória da espécie $i$ é dada por

$$
\mu_{i}\left(x_{i}\right)=\frac{\overline{\mu_{i}}}{1+e^{\beta\left(z-x_{i}\right)}},
$$

para $i=1,2 ; 0 \leqslant \bar{\mu}_{i} \leqslant 1$ é a fração migratória máxima específica para cada espécie e $\beta$ determina se a fração de migração é crescente ou decrescente.

Para o sistema em (2) e com as funções de migração e de dinâmica local como em (3) e (4), investigamos a formação de cluster na região em que $L_{T}>1$, com relação à segunda espécie. Nossas simulações evidenciam que o parâmetro $\beta$ tem influência direta sobre uma maior ou menor ocorrência desse fenômeno. Para ilustrar esse fato, consideramos uma rede de 10 sítios globalmente acoplados e analisamos as densidades populacionais da segunda espécie com relação aos parâmetros $r_{1}$ e $\beta$, mantendo os outros parâmetros fixados. Mais precisamente, escolhemos $r_{2}=3.7, \alpha=0.6$ e $r_{1} \in[5.4,6], \bar{\mu}_{1}=0.1$ e $\bar{\mu}_{2}=0.9$. É importante observar que não sabemos a priori quais grupos de sítios estarão sincronizados, logo a escolha das condições iniciais em nossas simulações é basicamente aleatória. A Figura 1(a) mostra a região de impossibilidade de

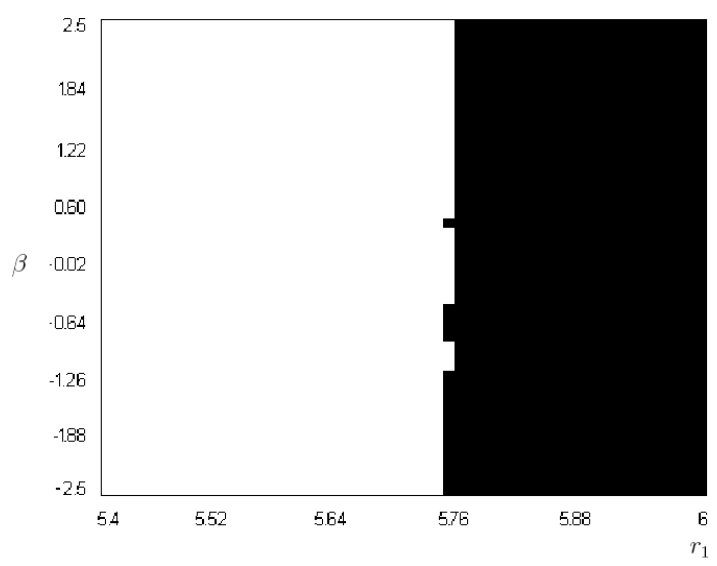

(a)

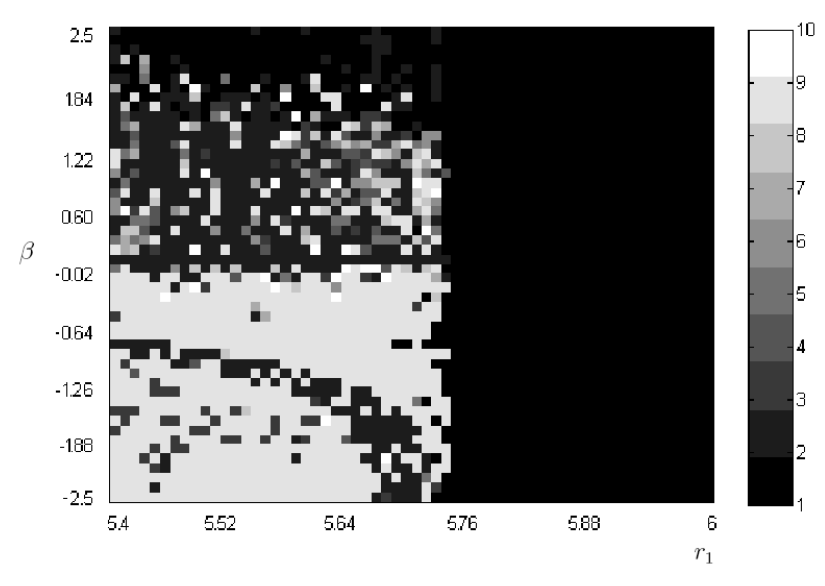

(b)

Figura 1: Diagrama de cores $r_{1}$ versus $\beta$ : (a) Região escura: $L_{T} \leq 1$; Região branca: $L_{T}>1$. (b) Formação de "clusters"para a segunda espécie.

coerência (região branca) em função dos parâmetros $r_{1}$ e $\beta$; a Figura 1(b) mostra a formação de "clusters" para a espécie dois, nessa mesma região. Utilizamos um padrão de cores em tons de preto a branco numa escala de 1 a 10, essa variação corresponde ao número de "clusters"que se formam para os parâmetros considerados, isto é, o número de grupos de sítios $n_{1}, \ldots, n_{J}$ como descritos anteriormente. Somente a região branca foi analisada, com isso podemos observar que a migração dependente da densidade exerce uma importante influência na dinâmica da espécie dois, já que a formação de "clusters" na região de impossibilidade de coerência depende de forma sensível das variações do parâmetro $\beta$. Esse estudo é biologicamente relevante, já que, apesar de estarmos considerando uma região onde os parâmetros são tais que não há a possibilidade de sincronização de ambas as espécies (como definido na Seção 2), uma delas pode sincronizar de forma total ou parcial, aumentando sua probabilidade de extinção. Podemos analisar o comportamento da dinâmica ilustrada na Figura 1 mais localmente, escolhendo $r_{1}=5.58$ e mantendo os mesmos valores para os outros parâmetros. A Figura 2 mostra a influência do parâmetro $\beta$ na formação de "clusters" para a espécie dois. Observamos que valores de $\beta>0$ parecem fornecer um maior espectro de possibilidades do que para $\beta<0$. Como ilustração, a Figura 3(a) mostra a série temporal da dinâmica da espécie dois para $\beta=0.8399$ e considerando todos os sítios. Podemos notar nessa figura o comportamento de dois grupos distintos, embora existam três 


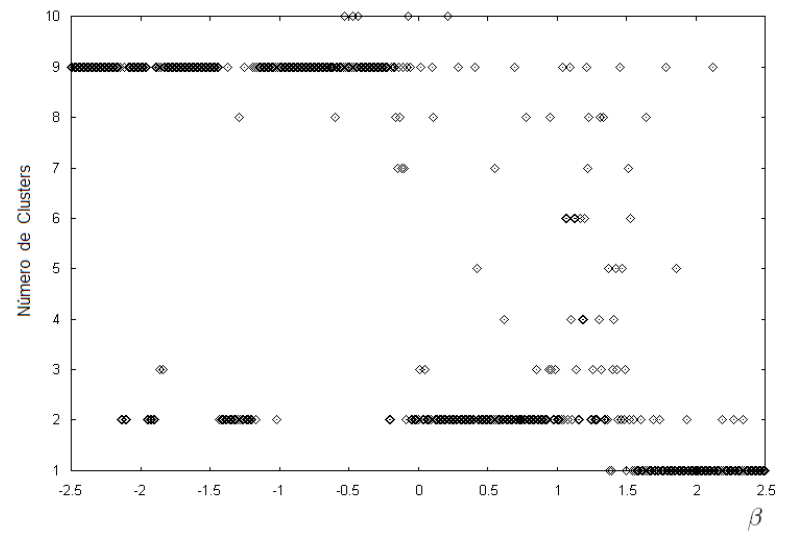

Figura 2: Número de clusters para a espécie dois em função do parâmetro $\beta$.

deles, sendo que a diferença absoluta entre dois deles é pequena e tem comportamento complexo do tipo on-off (ver Figura 3(b)). A Figura 2 nos fornece também indícios de que, para valores

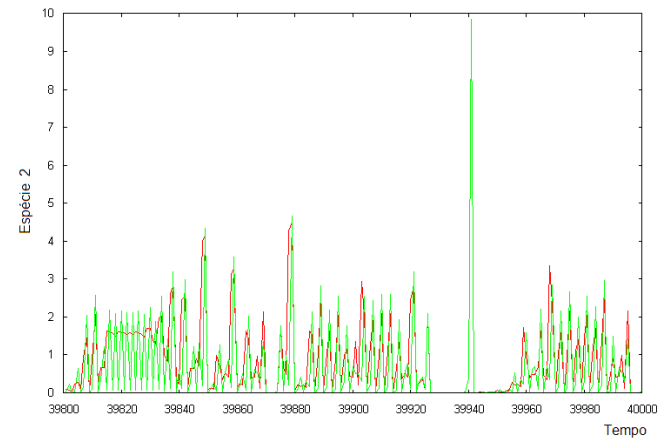

(a)

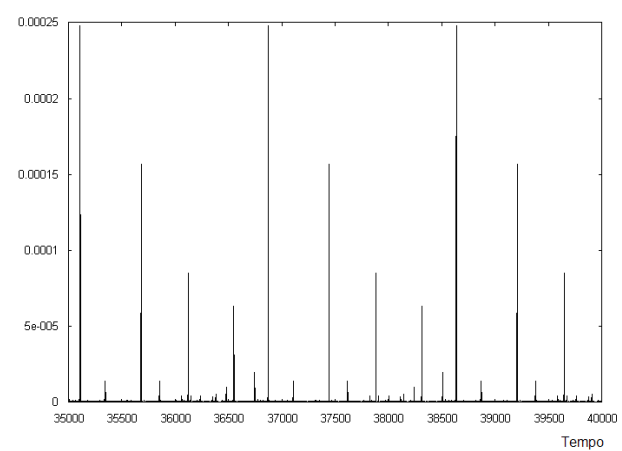

(b)

Figura 3: (a) Série temporal da espécie dois. (b) Diferença absoluta entre a população da espécie dois no sítio 1 e a do sítio 2 . Parâmetros: $\beta=0.8399, r_{1}=5.58, \alpha=0.6, r_{2}=3.7, \bar{\mu}_{1}=0.1 \mathrm{e}$ $\bar{\mu}_{2}=0.9$.

de $\beta$ em $[1.5,2.5]$, ocorre a formação de um único "cluster"para a espécie dois. No entanto, a espécie um é assíncrona, o que é razoável já que estamos considerando parâmetros na região de impossibilidade de coerência. Com isso, há a possibilidade de extinção de uma das espécies e permanência de uma delas. De fato isso ficou evidenciado em nossas simulações, como pode ser observado na Figura 4(a), onde a série temporal para a espécie dois (em todos os sítios) foi graficada; a Figura 4(b) mostra o erro de sincronização da espécie um, para esse mesmo valor de $\beta$, ficando evidente a extinção da espécie 2 e a pemanência da espécie 1 , cuja densidade populacional oscila de forma caótica.

O comportamento dos parâmetros $\bar{\mu}_{1}$ e $\bar{\mu}_{2}$ também parece induzir a formação de "clusters", na região de impossibilidade de coerência. Para o caso em que $\beta=1.5$, se os valores de $\bar{\mu}_{1}$ forem próximos de zero, a sincronização da espécie dois ocorre para valores intermediários ou pequenos de $\bar{\mu}_{2}$ (ver Figuras 5 (a) e (b)). Já para o caso de valores negativos de $\beta$, a região de impossibilidade de coerência é menor e a sincronização da espécie dois nessa região ocorre esparsamente; ela aparece, por exemplo, para valores de $\bar{\mu}_{1}$ e $\bar{\mu}_{2}$ pequenos (ver Figuras 6 (a) e (b)). 


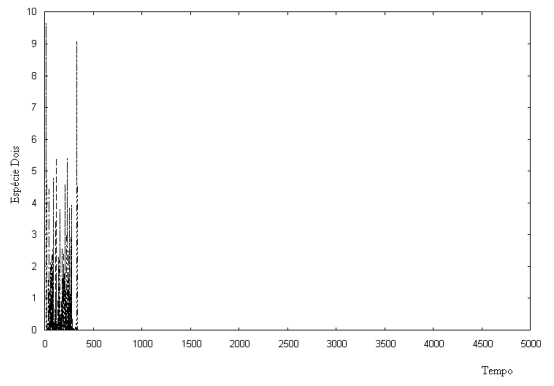

(a)

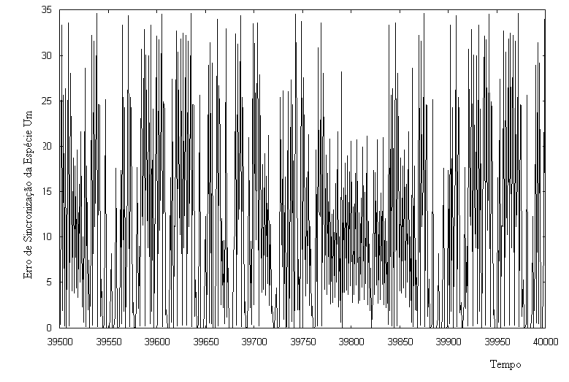

(b)

Figura 4: (a) Série temporal da espécie dois. (b) Erro de sincronização da espécie um. Parâmetros: $\beta=2.01, r_{1}=5.58, \alpha=0.6, r_{2}=3.7, \bar{\mu}_{1}=0.1$ e $\bar{\mu}_{2}=0.9$.

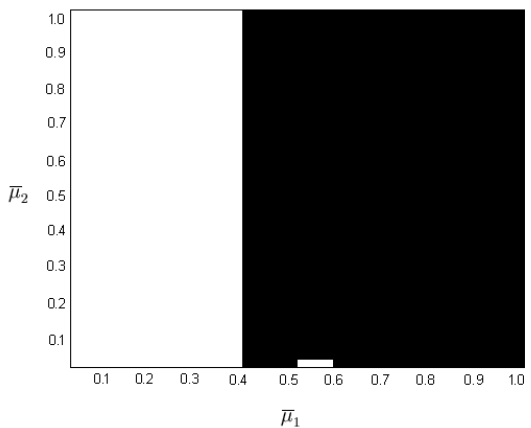

(a)

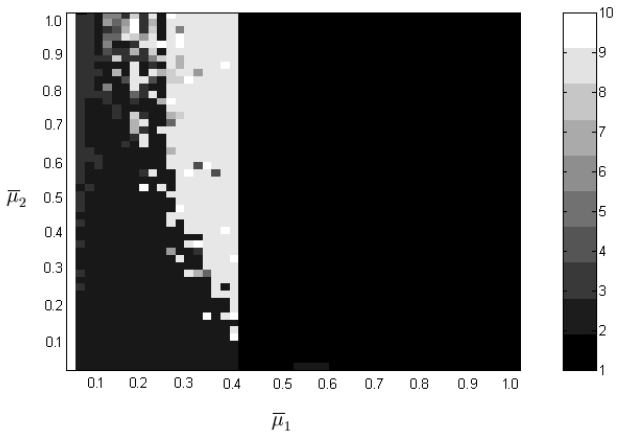

(b)

Figura 5: (a) Região em preto: $L_{T} \leq 1$. Região branca: $L_{T}>1$. (b) Formação de "clusters". Parâmetros: $\alpha=0.6, r_{2}=3.7, r_{1}=5.58$ e $\beta=1.5$.

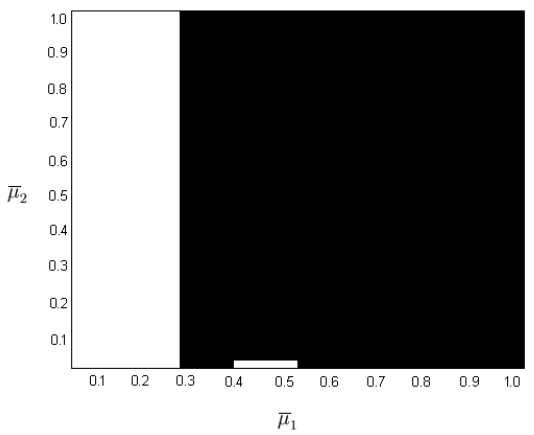

(a)

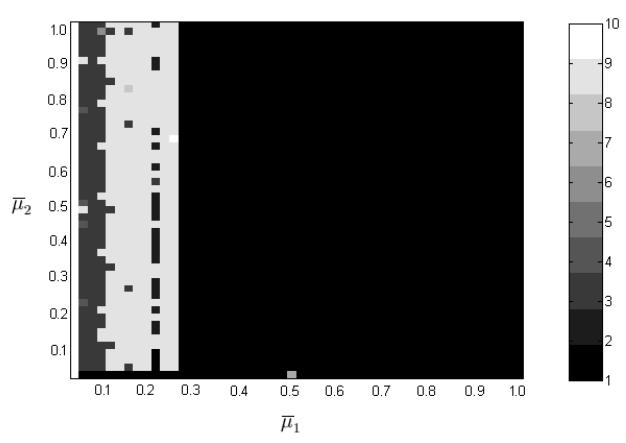

(b)

Figura 6: (a) Região em preto: $L_{T} \leq 1$. Região branca: $L_{T}>1$. (b) Formação de clusters. Parâmetros: $\alpha=0.6, r_{2}=3.7, r_{1}=5.58$ e $\beta=-1.5$. 


\section{Considerações Finais}

Apresentamos evidências numéricas de ocorrência de sincronização parcial e sua relação com os parâmetros da função de migração em um modelo metapopulacional de múltiplas espécies. Do ponto de vista biológico, para um modelo com hierarquia, nossos resultados ilustram que a migração dependente da densidade pode induzir sincronia total ou parcial de uma espécie enquanto outra tem comportamento caótico. Como consequência, uma das espécies pode ser extinta enquanto outra se conserva. Rigorosamente não podemos concluir que a tendência de formação de clusters que detectamos é decorrência da estabilidade assintótica transversal de atratores correspondentes a estados de sincronia parcial, pois as condições iniciais escolhidas podem não ser perturbações transversais às órbitas correspondentes. Não obstante esse fato, como descartamos os transientes e os resultados mostram-se muito similares para diferentes escolhas de pontos iniciais, suspeitamos que tais atratores tem bacias de atração grande, possivelmente do tipo riddled [3]. Estudos mais aprofundados de tais aspectos exigem a análise da dinâmica de perturbações transversais a subvariedades invariantes gerais [1], o que corresponde a futuras linhas de investigações.

\section{Referências}

[1] P. Ashwin, J. Buescu e I. Stewart, From attractor to chaotic saddle: a tale of transverse instability. Nonlinearity, 9 (1996), 703-737.

[2] J. Best, C. Castillo-Chavez e A. A. Yakubu, Hierarchical Competition in Discrete Time Models with Dispersal, Fields Institutional of Communications, 36 (2003), 59-86.

[3] B. Cazelles, S. Bottani e L. Stone, Unexpected coherence and conservation, Proc. R. Soc. Rev. E, 64 (2001) 2595-2602.

[4] D. J. D. Earn, S. A. Levin, P. Rohani, Coherence and Conservation, Science, 290 (2000), 1360-1364.

[5] F. T. Giordani, J. A. L. Silva, Sincronismo em um modelo metapopulacional com hierarquia e acoplamento não estacionário, Anais do XXXIII CNMAC, 3 (2010), 666-672.

[6] Gonzalez A, Lawton J H, Gilbert F S, Blackburn T M, Evans-Freke I., Metapopulation dynamics, abundance, and distribution in a microsystem, Science, 281 (1998), 2045-2047.

[7] R. A. Ims, H. P. Andreassen, Density-dependent dispersal and spatial populaton dynamics, Proc. Roy. Soc. B., 272 (2005),913-918.

[8] K. Kaneko, Relevance of dynamic clustering to biological networks, Physica D, 197 (1994), $55-73$.

[9] A. Pikovsky, M. Rosenblum e J. Kurths, "Synchronization: a universal concept in nonlinear sciences", Cambridge University Press, Cambridge, 2001.

[10] O. Popovych, Y. Mainstrenko e E. Mosekilde, Loss of coherence in a system of globally coupled maps, Physical Review E, 64 (2001), 1-11.

[11] J. A. L. Silva e F. T. Giordani, Density-dependent Migration and Synchronism in Metapopulations. Bull. Math. Biol., 68 (2006), 451-465. 